\title{
Metabolism as master of hematopoietic stem cell fate
}

\author{
Kyoko Ito ${ }^{1,2} \cdot$ Massimo Bonora ${ }^{1,2} \cdot$ Keisuke Ito ${ }^{1,2,3}$
}

Received: 12 August 2018 / Accepted: 10 September 2018 / Published online: 15 September 2018

(c) The Japanese Society of Hematology 2018

\begin{abstract}
HSCs have a fate choice when they divide; they can self-renew, producing new HSCs, or produce daughter cells that will mature to become committed cells. Technical challenges, however, have long obscured the mechanics of these choices. Advances in flow-sorting have made possible the purification of HSC populations, but available HSC-enriched fractions still include substantial heterogeneity, and single HSCs have proven extremely difficult to track and observe. Advances in single-cell approaches, however, have led to the identification of a highly purified population of hematopoietic stem cells (HSCs) that make a critical contribution to hematopoietic homeostasis through a preference for self-renewing division. Metabolic cues are key regulators of this cell fate choice, and the importance of controlling the population and quality of mitochondria has recently been highlighted to maintain the equilibrium of HSC populations. Leukemic cells also demand tightly regulated metabolism, and shifting the division balance of leukemic cells toward commitment has been considered as a promising therapeutic strategy. A deeper understanding of precisely how specific modes of metabolism control HSC fate is, therefore, of great biological interest, and more importantly will be critical to the development of new therapeutic strategies that target HSC division balance for the treatment of hematological disease.
\end{abstract}

Keywords Cellular metabolism $\cdot$ Mitochondria $\cdot$ Hematopoietic stem cell $\cdot$ Leukemia $\cdot$ Stem cell fate

\section{Introduction}

Hematopoiesis is the process by which the various cellular components of the blood are formed. The short-term need for these components can be highly variable, but evidence has accumulated that hematopoietic stem cells (HSCs) are capable of meeting even drastic changes in demand through a variety of differentiation paths. Like other stem cells, HSCs are self-renewing, and either multi- or uni-potent [1-5]; these unique capacities offer opportunities for stem cell-based therapies [6] which can be tested via transplantation experiments. To this end, considerable progress has

Keisuke Ito

keisuke.ito@einstein.yu.edu

1 Ruth L. and David S. Gottesman Institute for Stem Cell and Regenerative Medicine Research, Albert Einstein College of Medicine, Bronx, NY, USA

2 Departments of Cell Biology and Medicine, Albert Einstein College of Medicine, Bronx, NY, USA

3 Albert Einstein Cancer Center and Diabetes Research Center, Albert Einstein College of Medicine, Bronx, NY, USA been made over the last three decades in purifying populations of HSCs as well as the different downstream multipotent progenitors (MPPs), and the uni-lineage progenitors found in humans and rodents [7]. Fluorescence-activated cell sorting (or FACS) greatly facilitated the purification of transplantable HSCs through positive and negative selection, as described in pioneering publications (e.g., [8] from Dr. Irving Weissman's group). HSCs are widely believed essential to hematopoiesis under recovery or other stress conditions [9-13], and HSC transplantation has, therefore, been a key therapeutic strategy in combatting hematological disorders [14, 15]. Intriguingly, recent advances utilizing single-cell approaches have revealed at the apex of hematopoiesis a rare population of HSCs that can sustain all the hematopoietic lineages throughout the lifetime of the individual, and may be critical to the rapid hematopoietic response required by acute conditions.

Isolation of relatively pure populations of cells of defined functionality has also enabled the analysis of the cellular basis of normal hematopoiesis, which has proved critical in advancing our understanding of how the malignant cells of hematologic diseases arise from 
normal hematopoietic cells through genetic and epigenetic changes. Like the stem cells of other tissues, HSCs basically remain quiescent to maintain their undifferentiated state [2, 3]. Deletion of the genes involved in quiescence often leads to HSC exhaustion due to uncontrolled proliferation [16-21], and the regenerative potential of HSCs may be governed by their divisional history $[2,3]$. HSC self-renewal capacity is fine-tuned by cell intrinsic networks in concert with the activity of transcriptional factors; together these cooperate with cumulative signals from the microenvironment to maintain whole hematopoiesis [17, 19, 22-26]. The metabolic cues involved in regulating HSC self-renewal capacity have, therefore, become the focus of much current research, which has already yielded many significant insights [27-33] (Fig. 1). In this review, we will highlight the recent advances in our understanding of the intriguing relationship between cellular metabolism, mitochondrial quality control, and HSC fate decisions. Finally, we will also discuss the current and future challenges faced by researchers seeking to identify the key metabolic pathways maintaining stemness upon division of leukemia stem cells (LSCs), as well as the therapeutic promise of new strategies targeting leukemia metabolism.

\section{Hematopoietic differentiation tree: HSCs and their downstream populations}

After critical advances in the characterization of progenitor populations downstream of HSCs, a consensus model emerged of the hematopoietic differentiation tree [34]. In this model, the first branch point segregates lymphoid potential from all other lineages (myeloid, erythroid and megakaryocytic), followed by further branching steps on either side of the tree progressing from multi- to bi- and finally to uni-potent progenitor cells. HSC pools are divided into long-term HSCs (LT-HSCs), which provide long-term engraftment and can serially engraft irradiated mice, and short-term HSCs (ST-HSCs), which have more limited selfrenewal capacity in a serial transplantation assay.

A

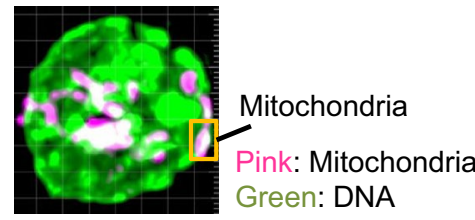

B

Mitochondrial function

- Cellular metabolisms

- Bioenergetics

Electrons from reducing equivalents feed into ETC to generate ATP

- Biosynthesis Intermediary metabolites of TCA cycle are used to synthetize macromolecules (e.g. lipids, carbohydrates, proteins and nucleoties)

- Signaling organelle e.g. $\mathrm{ROS}$ release, $\mathrm{Ca}^{+}{ }^{+}$homeostasis

- Regulation of cell death

- Estrogen synthesis

- Heme synthesis

Fig. 1 Key metabolic pathways in hematopoietic stem cells. a Representative deconvoluted immuno-fluorescent images of mitochondria (top) and mitochondrial functions (bottom). Mitochondria are important bioenergetic and biosynthetic organelles, and are also involved in cell signaling pathways. The reducing equivalents generated by the tricarboxylic acid (TCA) cycle are fed to the electron transport chain

(ETC) to drive the synthesis of ATP molecules. b Hematopoietic stem cells (HSCs) rely on glycolysis, which is promoted by hypoxiainducible factor $1 \alpha$ (HIF-1 $\alpha)$. Valine, one of the essential amino acids (EAAs), is required for the proliferation and maintenance of HSCs. The intermediate of the TCA cycle, $\alpha$-ketoglutarate $(\alpha \mathrm{KG})$, is a cofactor for dioxygenase enzymes 
Beyond this basic classification, however, the pathways of differentiation remain under debate. HSCs are believed to give rise to an MPP that is capable of producing all blood cell lineages but lacks the capacity for serial transplantation in mice. This MPP then differentiates into the myeloid and lymphoid lineages. The common myeloid progenitor (CMP) gives rise to all myeloid cells. Similarly, the common lymphoid progenitor (CLP) can differentiate into B and $\mathrm{T}$ lymphocytes and natural killer (NK) cells. The CMP further differentiates into two progenitors with more restricted differentiation potential, the megakaryocyte-erythroid progenitor (MkEP) and a granulocyte-macrophage progenitor (GMP).

The subsequent identification of other surface markers has suggested several modifications of this classical tree. Lymphoid and myeloid fates now remain linked prior to separation further down the tree, while megakaryocyte branching arrives earlier, along with subdivision of the MPP compartment into distinct subpopulations. Other aspects of the model are being reconsidered: the first lineage commitment step from the HSC pool, for instance, may not be a lymphoid-myeloid differentiation decision step but rather one that allows the megakaryocyte-erythroid lineage to split off, leaving a progenitor with both lymphoid and myeloid (granulocyte-macrophage) potential. Termed a lymphoid primed multipotential progenitor (LMPP), this cell then differentiates into the granulocyte-macrophage and lymphoid lineages. T-cell/myeloid progenitors and B-cell/myeloid progenitors have now been isolated, and there is mounting evidence that cell intermediates with combined myeloid/ lymphoid potential may exist. Moreover, the existence of mixed myeloid/lymphoid leukemias supports the notion that there may be a normal counterpart of this malignant population [7, 35].

Further evidence suggests that the picture may be even more complicated, as the HSC pool itself is functionally and molecularly heterogeneous. HSCs have been identified retrospectively after single-cell transplantation by clonal assays, and these assays have demonstrated the heterogeneity of currently available HSC-enriched fractions. Using limiting-dilution analysis and single-cell transplantation, a research team led by Dr. Connie Eaves has described HSCs that differ in their relative myeloid and lymphoid output [36, 37]. Groups led by Drs. Sten Erik Jacobsen and Hiromitsu Nakauchi recently identified platelet-biased HSCs that predominantly differentiate toward megakaryocytes and platelets, and subsequent transplantation experiments have revealed that single HSCs execute only a limited repertoire of lineage fate patterns, with platelets providing the only observed long-term unilineage read-out [38-40]. Dr. Nakauchi's lab further proposed an additional differentiation model in which HSCs can directly differentiate into lineage-restricted progenitors while bypassing the multipotent progenitor stage during acute conditions that demand rapid replenishment of mature cells (as in response to ablation stress) [40]. Notably, phenotypic HSCs comprise a major source of the megakaryocyte/platelet lineage in steady-state conditions, but these cells show "multi"-lineage differentiation capacity once they are transplanted into irradiated recipient mice $[13,41]$. These data imply potential differences in fate decision mechanisms between steady state and hematopoietic recovery. Indeed, theories differ regarding the contributions of HSCs to unperturbed homeostasis vs. tissue recovery conditions, and technical considerations may have an impact on conclusions derived from transplantation experiments.

Although it is likely that all these fate decision models capture true aspects of HSC differentiation, in aggregate they would be difficult to squeeze into a single, rigidly branching tree. New ways of graphically representing the process of HSC differentiation are, therefore, required. Recent advances in single-cell transcriptomic snapshots have suggested a new model of differentiation in which acquisition of lineage-specific fates is a continuous process, and unilineage-restricted cells emerge directly from a continuum of low-primed undifferentiated hematopoietic stem and progenitor cells (HSPCs), without major transitions through the multi- and bi-potent stages. It is also clear that genetic changes (such as mutations) or epigenetic changes, either induced by experiments or derived from disease states, can alter the progress of cells through these compartments. This new continuum model is based on single-cell transcriptomic data, however; the biological validation of a model in which all lineages branch directly from the HSC compartment would require additional supportive evidence.

\section{Metabolic control in HSC homeostasis and fate decision}

HSCs maintain both their multipotentiality and self-renewal capacity throughout the life of the organism, and once specified, they have the option of progressing down any one of a number of cell-fate paths. Most of the time, however, HSCs are quiescent and remain in $G_{0}$ of the cell cycle, although they continue to be capable of undergoing cell divisions as required. As alterations in the equilibrium of HSC selfrenewal and commitment can affect hematopoietic homeostasis, the mechanisms of division balance are clearly critical to HSC maintenance; however, the means for readily studying the functional symmetry of individual pairs of daughter cells have so far been lacking. In contrast, assessment of populations of paired daughter cells has proved a powerful tool in evaluating the self-renewal capacity of HSCs by analyzing cells both qualitatively and quantitatively after they have divided [16, 40-43]. The possible division patterns of 
HSCs are symmetric division (SD), asymmetric division (AS) and symmetric commitment (SC), and pattern choice is determined by either the in vivo repopulation capacity or the in vitro colony-forming capacity of the daughter cells in question.

Several groups have directly evaluated the homogeneity of HSC population by both the multi-lineage repopulation and HSC regeneration capacities of individual cells purified from HSC fractions. These studies have shown that the general frequency of HSCs in these fractions is only one in several cells [36, 40, 44, 45]. Unfortunately, this functional heterogeneity masks the behavior of individual cells within the fraction because any contamination by non-HSCs can lead to an overestimate of the rate of SC, which causes difficulty in identifying HSC-specific phenotypes or elucidating the division patterns of pure HSC populations [43]. To address these problems, researchers have developed single-cell tracking methods using reporter mouse strains. However, most of these efforts have been undertaken in lessenriched HSPC populations, or did not include functional validation by in vivo transplantation assay at the single-cell level [10, 38, 39, 46-49]. To establish a single-cell assay system with individual HSCs, and then visualize their divisions, it is critical to identify specific marker(s) that enable isolation of a highly purified fraction of HSCs.

Our validation of Tie 2 positivity as a marker has allowed us to identify a purified population of HSCs using Tie 2 reporter combined with antibody-mediated purification

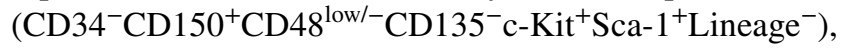
and we have demonstrated with our own local transplantation protocol that this population is of far higher purity than HSC fractions previously reported, with minimal heterogeneity within the fraction $[50,51]$. We have further shown that Tie 2 positivity is strongly associated with repopulation capacity. Our tracking technique allowed us to determine the function of single pairs of daughter cells resulting from the divisions of single $\mathrm{Tie}^{+} \mathrm{HSCs}$, which in turn enabled us to more accurately visualize division patterns, and distinguish self-renewal expansion from self-renewal maintenance. Our findings demonstrated that most of the initial divisions of $\mathrm{Tie}^{+}$HSCs were found to be symmetrical divisions, with both daughter cells retaining Tie 2 positivity and repopulation capacity at the single-cell level [50].

The high self-renewal capacity of $\mathrm{Tie}^{+} \mathrm{HSCs}$ led us to explore their regulatory mechanisms, in the hope of eventually enabling the manipulation of factors regulating division patterns and self-renewal. We subsequently identified mitophagy, a quality control process of the mitochondria, as a key mechanism of self-renewing HSC expansion (Fig. 2). Our single-cell gene expression assays revealed that a substantial number of genes are expressed at higher levels in $\mathrm{Tie}^{+}$than Tie $2^{-} \mathrm{HSCs}$; these include HSC factors/markers as well as signaling pathways regulating fatty acid oxidation
(FAO) via carnitine palmitoyltransferase $1 \mathrm{a}(\mathrm{Cpt} 1 \mathrm{a})$ and peroxisome proliferator-activated receptor delta (Ppard). Hierarchical clustering and signatures for FAO likewise demonstrated that $\mathrm{Tie}^{+} \mathrm{HSCs}$ are molecularly distant from both Tie $2^{-}$HSCs and progenitor cells [50].

Replicative stress by the activation of dormant HSCs is known to lead to the accumulation of DNA damage, toxic metabolites and dysfunctional mitochondria, and the eventual loss of stem cellness [17, 19, 52]. Notably, in asymmetric division, defective mitochondria and unwanted organelles can be separated from the stem cell daughters yielded by the division and consigned to the differentiated daughter cells [53], but in the case of symmetric self-renewing division, damaged organelles are apparently disposed of by both daughter cells, as young and old mitochondria have been found equally distributed between both $[30,50]$. This suggests that some unidentified level of defective organelles may represent a limiting factor on self-renewing division, and indeed, it has proved very challenging to expand the HSC population while maintaining stemness through symmetric division by the manipulation of a single factor, particularly given that the mechanics of increasing self-renewal capacity have so far remained unknown.

We have recently shown, however, that cellular metabolism is critical to HSC expansion by demonstrating at the single-cell level that the defective self-renewal capacity of Promyelocytic leukemia $(\mathrm{Pml})$ knockout $\mathrm{Tie}^{+}$HSCs can be rescued by pharmacological PPAR-FAO activation and its ensuing promotion of mitophagy [50]. Mitochondrial autophagy, or mitophagy, is a specific form of autophagy for the selective clearance of damaged mitochondria [54], and two Parkinson's disease genes, mitochondrial kinase Pteninduced putative kinase 1 (Pink1) and ubiquitin ligase Parkin (or Park2), play key roles in its regulation (Fig. 2a) [54]. Activation of PPAR-FAO led to expanded Tie $2^{+} \mathrm{HSC}$ populations through enhanced Parkin recruitment in the mitochondria. Conversely, inhibition of mitophagy by acutely silencing Parkin attenuated HSC expansion (Fig. 2b). These metabolic pathways were found to be conserved and operational in human HSCs. Our data thus identify a clear link between symmetric division and the self-clearance systems found in both daughter cells of HSCs, and suggest potential strategies of HSC fate manipulation through key metabolic pathways [50].

Mitochondria are bioenergetic hotspots, and produce the bulk of ATP by the oxidative phosphorylation process (Fig. 1). HSCs exhibit lower levels of both baseline and maximal respiration than progenitor cells, and proper control of mitochondrial function is a key factor in HSC maintenance [29]. HSCs are kept in as quiescent a state as possible to avoid losing their repopulating capacity, and, like other tissue stem cells, rely on glycolysis in this state rather than mitochondrial oxidative phosphorylation (Oxphos). 


\section{A}

\section{Mitochondrion level}

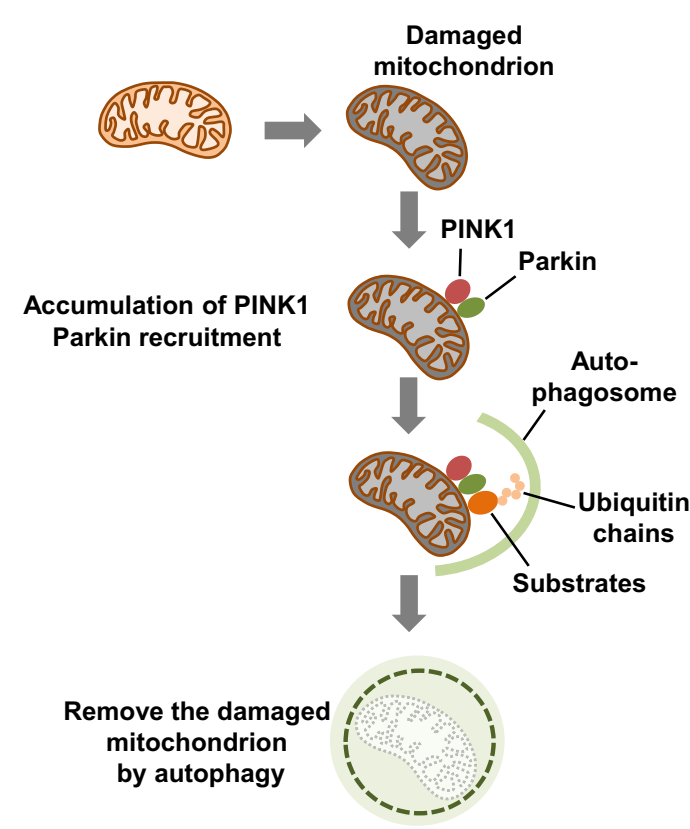

Fig. 2 Quality control of mitochondria promotes HSC self-renewal. a The mechanisms of mitophagy, a selective removal process of mitochondria, has been extensively explored, and one of the best-understood pathways in this process is Pink1/Parkin-mediated mitophagy. In healthy mitochondria, Pink1 is imported and constitutively degraded. In depolarized mitochondria, Pink1 (red) is stabilized on the outer mitochondrial membrane and facilitates the recruitment of cytosolic Parkin (green). Activation of Parkin leads to the ubiquitina-

Enhanced respiration is detrimental to HSC maintenance and function [20, 55-65], and cell cycle entry elevates levels of intracellular reactive oxygen species (ROS) through the activation of Oxphos. As quiescent HSCs are generally sensitive to increased intracellular ROS, repeated cell divisions lead to reduced self-renewal capacity and, ultimately, HSC exhaustion [27, 28, 66-72]. For example, recent studies from Dr. Danica Chen's group have shown that the deletion of a histone deacetylase Sirtuin 7 (Sirt7) increased mitochondrial protein folding stress $\left(\mathrm{PFS}^{\mathrm{mt}}\right)$ in HSCs through activation of nuclear respiratory factor (Nrf1). The disruption of this metabolic checkpoint led to the loss of HSC quiescence and reduced regenerative capacity with myeloid-biased differentiation. The expression of Sirt7 is reduced in older HSCs, suggesting that its downregulation contributes to the functional decline of aging HSCs. Indeed, re-introduction of Sirt7 in aged HSCs reduces PFS ${ }^{\mathrm{mt}}$ and improves their regenerative capacity $[73,74]$. Once differentiated, HSCs must switch to mitochondrial metabolism to meet rapidly increasing energy demands. PTPMT1 encodes a PTEN-like
B

\section{Cellular level}

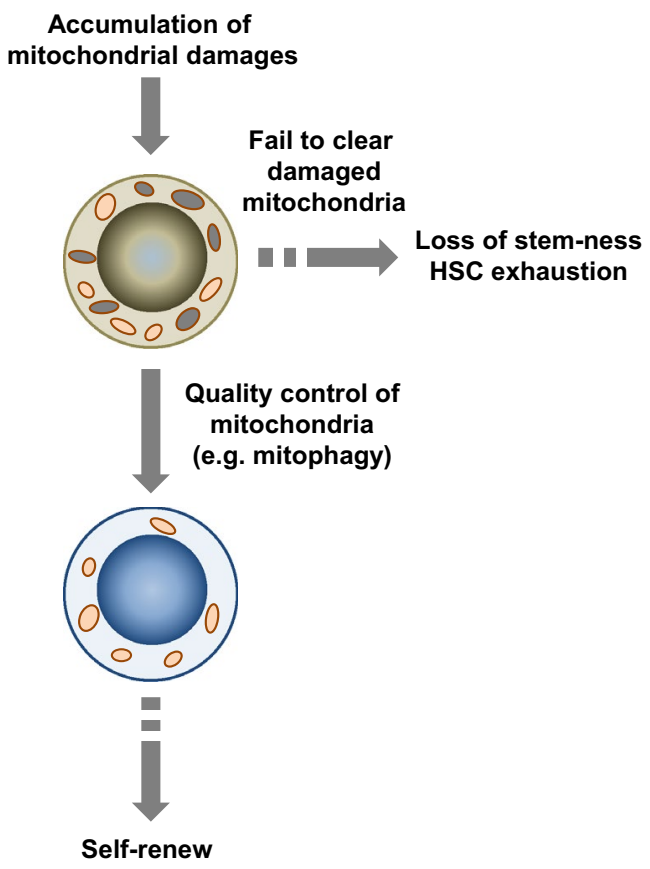

tion (orange) of multiple outer protein substrates, which are recognized by specific mitophagy receptors. Phagophore (pale green) surrounds the damaged mitochondrion, which is subsequently delivered to the lysosomal clearance. b Upon cell division, organelles such as mitochondria may be damaged, which promotes mitochondrial quality control, which in turn supports the self-renewal of HSCs. Failure to clear the damaged mitochondria could lead to HSC exhaustion

mitochondrial phosphatase, and the inhibition of mitochondrial respiration by conditional knockout of Ptpmt1 impaired HSC differentiation, while HSC population is drastically increased [75].

The mitochondria may play significant roles in cell fate choice beyond the process of Oxphos. Increasing evidence indicates that mitochondria contribute to the preservation of HSC self-renewal capacity by linking cellular metabolism and epigenetics [76-80]. Furthermore, a mitochondrial fusion regulator, mitofusin-2 (Mfn2), which is induced by the short isoform of PR domain containing 16 (Prdm16), is required for HSC maintenance, and is especially critical to cell differentiation to the lymphoid lineage. This is mediated by intracellular calcium buffering provided by the tethering activity of mitochondria to the endoplasmic reticulum [81, 82]. Interestingly, it has also been shown that increased intracellular $\mathrm{Ca}^{2+}$ levels in HSCs drive cell divisions, and the appropriate suppression of $\mathrm{Ca}^{2+}$ levels by exogenous adenosine has been identified as an important regulator of HSC fate [83]. 
As autophagy has been shown to have an indirect but significant effect on HSC metabolism, researchers have begun to explore the roles of macroautophagy (or simply autophagy) in hematopoiesis and HSC aging [84-89]. In autophagy, cytoplasmic contents are sequestered into the double-membrane autophagosome, which fuses with the lysosome to become the autolysosome. Autophagy-related (Atg) conjugation systems are a crucial element in this delivery process, along with the lysosomal degradation pathway, which contributes to cellular quality control through the elimination of functionally impaired organelles and proteins. When $\operatorname{Atg} 7$ has been conditionally depleted in hematopoietic systems, the resulting defects in autophagy have led to damaged mitochondria and lethal anemia $[90,91]$.

A study from Dr. Emmanuelle Passegue's group recently used Atg12 deletion to illuminate the roles of autophagy in the health and longevity of HSCs [92]. One-third of HSCs from older mice exhibit high autophagy activity and a low metabolic state, and these cells maintain a regeneration potential similar to that of HSCs from young mice. When Atg 12 is conditionally deleted from HSCs, the impairment of autophagy leads to a skewed ratio of circulating myeloid versus lymphoid cells in primary mice, and reduced regenerative potential. These blood aging phenotypes are accompanied by epigenetic deregulations and elevated Oxphos. When HSCs enter into the cell cycle, metabolic activity shifts from glycolysis to mitochondrial Oxphos; these newly active HSCs are believed to soon return to glycolysis, however, to maintain their regenerative pool. Findings from studies of Atg12-conditional knockout mice suggest that autophagy plays an essential role in reverting metabolically active HSCs to quiescence by efficiently clearing healthy but active mitochondria, which prevents the induction of (epi) genetic programs that lead to HSC commitment, and thereby supports the maintenance of healthy hematopoiesis [92].

Important contributions of autophagy to hematopoiesis have also been identified by Dr. Hui-Kuan Lin's group in a study of genetic mouse models exhibiting hyper-activated mitophagy [93]. Atad3a (or ATPase family, AAA domain containing $3 \mathrm{~A}$ ) is a mitochondrial membrane protein which regulates dynamic interactions between the mitochondrial outer and inner membrane complexes. Dr. Lin's team demonstrated that Atad3a prevents accumulation of Pink1 by promoting its import into mitochondria for degradation [93]. Acute deletion of Atad3a was found to induce mitophagy of both healthy and damaged mitochondria, which blocked hematopoietic commitment at the progenitor stage and led to expanded HSPC pools. These phenotypes are at least in part mediated by Pink1, as Pink1 ablation reduced mitophagy levels and partially rescued the defective hematopoietic phenotypes caused by Atad3a ablation [93]. Collectively, these studies demonstrate that appropriate regulation of both selective and unselective autophagy is critical to hematopoietic homeostasis; the specific mechanics of the relationship between HSC fate and cellular metabolism, however, have only begun to be understood. A further characterization of metabolic requirements governing HSC fate decisions will undoubtedly lead to potential strategies for promoting HSC self-renewing division through metabolic manipulation.

\section{Key open questions: application to hematologic disorders}

A disturbed division balance causes hematological disorders [94, 95], and the long-term survival rate among blood cancer patients remains stubbornly low, as most patients who achieve remission eventually relapse. In hematologic malignancies, mutations are present in the genes regulating intracellular signaling. The presence of initiating mutations that promote clonal expansion, and thus a pre-malignant state, has long been suspected as a necessary precursor to the development of most malignancies.

Exome sequencing of peripheral blood samples from more than 30,000 patients without overt hematological malignancies demonstrated recurrent somatic myeloid malignancy-associated mutations in $\sim 10 \%$ of patients over the age of 65 and $>20 \%$ of patients over the age of 90 [96, 97]. This phenomenon has subsequently been termed clonal hematopoiesis of indeterminate potential (CHIP). The common recurrently mutated genes of this group are epigenetic modifiers, DNA methyltransferase 3A (DNMT3A), Teneleven translocation 2 (TET2) and additional sex combs-like 1 (ASXL1), all of which are also mutated in myelodysplastic syndrome (MDS) [96-98].

The absolute risk of transformation to overt malignancy in patients with CHIP was low during the time periods under study, and a diagnosis of CHIP requires the presence of a somatic mutation with a mutant allele fraction of at least $2 \%$ in the peripheral blood, with no other evidence of a hematological malignancy [99]. MDS is generally a more genetically complex disease than CHIP, with most patients harboring at least two, and sometimes many more, somatic mutations in recurrent driver genes, often with a high mutant allele fraction $(>10 \%)$, at the time of diagnosis. Mutations that occur early in disease evolution can be detected either by calculating allele frequency in bulk sequencing studies or by single-cell sequencing, and using these methods, several studies have demonstrated that splicing factors and epigenetic modifiers tend to be mutated early in the evolution of MDS, whereas mutations in transcription factors (RUNXI and GATA2) can be either early or late events [100].

Leukemia stem cells (LSCs, also known as leukemiainitiating cells) are believed to not only drive disease initiation, progression, and drug resistance, but also contribute to 
relapse [101-105]. Elimination of every single LSC is, therefore, essential to a long-term cure. Upon division, LSCs can either self-renew or commit to differentiation, and shifting their division balance away from renewal and toward commitment holds great promise as a therapeutic strategy [106, 107]. Research into possible targets for such an approach has already begun; for example, studies from multiple groups have demonstrated that Musashi-2 (MSI2), a molecule that binds RNA, increases proliferation of malignant blood stem cells. Msi2 is the predominant form expressed in HSCs, and its knockdown leads to reduced engraftment and depletion of HSCs in vivo. Conversely, ectopic overexpression of Msi2 in a transgenic model leads to an increased progression of cell cycle in HSPCs, with changes in asymmetric segregation of Numb. Importantly, increasing Msi1 expression can promote leukemic progression. In humans, MSI2 was found in large amounts in acute myeloid leukemia (AML) and chronic myeloid leukemia (CML), and high MSI2 expression predicted short survival. Indeed, Msi2 cooperated with $\mathrm{BCR}-\mathrm{ABL}$ to induce an aggressive acute leukemia comparable to CML-blast crisis [95, 107, 108].

Another study, again from Dr. Tannishtha Reya's group, uncovered a role for the cytoplasmic dynein-binding protein lissencephaly-1 (encoded by Lis1, also known as PafhalB1) in leukemogenesis [94]. Live-cell imaging upon division of HSPCs showed that spindle orientation determines the division plane, and thus the symmetric or asymmetric deposition of Numb upon division. When Lisl is deleted, both spindle rotation and inheritance of Numb are impaired. This change accelerated cell differentiation rates followed by rapid exhaustion of the HSPC pool. Importantly, the transformed hematopoietic cells in mouse models with AML rely on Lis1-mediated regulation of cell division during the clonal outgrowth of cells with differentiation blockade, which is a critical phase of leukemogenesis. Knockdown of LISI in human primary $\mathrm{CD} 34^{+}$cells resulted in impairment of leukemic growth.

\section{Conclusion and perspectives}

Increasing evidence has shown that specific modes of metabolism play important roles in the self-renewal capacity of both healthy and transformed stem cells. LSCs demand tightly regulated metabolism, since disruption of either glycolysis or mitochondrial respiration impairs leukemogenesis $[27,109-111]$. It is no surprise that the metabolic requirements of leukemogenesis and LSC function have, therefore, become highlighted.

Some preliminary findings have already hinted at therapeutic promise. For example, vitamin $\mathrm{C}$ has been found to mimic Tet 2 restoration in Tet2-knockdown HSCs by promoting DNA demethylation and reversing aberrant self-renewal, thereby altering the progression of leukemia $[80,112]$. HSCs are highly sensitive to valine, one of the three branched-chain amino acids (BCAAs), and it has been shown that dietary depletion of valine could potentially be used as a conditioning regimen for HSC transplantation [113]. Branched-chain aminotransferase 1 (BCAT1) converts BCAAs into branched-chain ketoacids by transferring the BCAA amino group onto alpha-ketoglutarate $(\alpha \mathrm{KG})$, a substrate for TET2; as it is overexpressed in TET2/Isocitrate dehydrogenase $(I D H)$ wild-type acute myeloid leukemia LSCs [76, 114]; it has, therefore, been proposed that BCAT1 may drive LSC self-renewal and leukemogenesis at least in part by reducing TET2 activity. IDH enzymes catalyze production of $\alpha \mathrm{KG}$, and mutant IDH enzymes catalyze production of 2-hydroxyglutarate, which leads to aberrant selfrenewal and leukemogenesis in part through the inhibition of TET2 activity. These are just few recent insights into LSC metabolism that may have clinical implications, and we are only beginning to understand the LSC metabolism.

The discovery of contributions to leukemogenesis by metabolism, mitochondrial biogenesis, and cytoprotective autophagy support the notion that mitochondrial quality control by autophagy may be a key determinant of division balance [115]. However, tracking the division pattern of individual LSCs has proved challenging, and the development of new single-cell assay techniques is critical to achieving a better understanding of the molecular basis of LSC fate choice [116, 117]. Identifying the key metabolic cues that control LSC fate and maintain stemness upon division could yield effective targets in strategies to enhance LSC commitment, and will, therefore, have clear clinical importans.

Acknowledgements We are grateful to members of the Ito lab and Einstein Stem Cell Institute for their comments on HSC self-renewal and metabolisms, and most importantly, to the organizing committee and Dr. Masahiro Kizaki for giving us a great opportunity to present our work at JSH 2017. Ke.I. is supported by grants from the National Institutes of Health (R01DK98263, R01DK115577, and R01DK100689), New York State Department of Health as Core Director of Einstein Single-Cell Genomics/Epigenomics (C029154). Ke.I. is a Research Scholar of the Leukemia and Lymphoma Society. We apologize to the investigators whose work could not be cited owing to space limitations.

\section{References}

1. McCulloch EA, Till JE. The radiation sensitivity of normal mouse bone marrow cells, determined by quantitative marrow transplantation into irradiated mice. Radiat Res. 1960;13:115-25.

2. Weissman IL, Anderson DJ, Gage F. Stem and progenitor cells: origins, phenotypes, lineage commitments, and transdifferentiations. Annu Rev Cell Dev Biol. 2001;17:387-403. https://doi. org/10.1146/annurev.cellbio.17.1.387.

3. Visvader JE, Clevers H. Tissue-specific designs of stem cell hierarchies. Nat Cell Biol. 2016;18:349-55. https://doi.org/10.1038/ ncb3332. 
4. Morrison SJ, Shah NM, Anderson DJ. Regulatory mechanisms in stem cell biology. Cell. 1997;88:287-98.

5. Ramalho-Santos M, Willenbring $H$. On the origin of the term "stem cell". Cell Stem Cell. 2007;1:35-8. https://doi. org/10.1016/j.stem.2007.05.013.

6. Daley GQ, Goodell MA, Snyder EY. Realistic prospects for stem cell therapeutics. Hematol Am Soc Hematol Educ Progr. 2003;2003:398-418.

7. Laurenti E, Gottgens B. From haematopoietic stem cells to complex differentiation landscapes. Nature. 2018;553:418-26. https://doi.org/10.1038/nature25022.

8. Spangrude GJ, Heimfeld S, Weissman IL. Purification and characterization of mouse hematopoietic stem cells. Science. 1988;241:58-62.

9. Sun J, et al. Clonal dynamics of native haematopoiesis. Nature. 2014;514:322-7. https://doi.org/10.1038/nature13824.

10. Busch K, et al. Fundamental properties of unperturbed haematopoiesis from stem cells in vivo. Nature. 2015;518:542-6. https://doi.org/10.1038/nature14242.

11. Sawai CM, et al. Hematopoietic stem cells are the major source of multilineage hematopoiesis in adult animals. Immunity. 2016;45:597-609. https://doi.org/10.1016/j.immun i.2016.08.007.

12. Ito K, Frenette PS. HSC contribution in making steady-state blood. Immunity. 2016;45:464-6. https://doi.org/10.1016/j. immuni.2016.09.002.

13. Rodriguez-Fraticelli AE, et al. Clonal analysis of lineage fate in native haematopoiesis. Nature. 2018;553:212-6. https://doi. org/10.1038/nature25168.

14. Appelbaum FR. Hematopoietic-cell transplantation at 50. N Engl J Med. 2007;357:1472-5. https://doi.org/10.1056/NEJMp 078166.

15. Copelan EA. Hematopoietic stem-cell transplantation. N Engl J Med. 2006;354:1813-26. https://doi.org/10.1056/NEJMra0526 38.

16. Ito $\mathrm{K}$, et al. A PML-PPAR-delta pathway for fatty acid oxidation regulates hematopoietic stem cell maintenance. Nat Med. 2012;18:1350-8. https://doi.org/10.1038/nm.2882.

17. Wilson A, et al. Hematopoietic stem cells reversibly switch from dormancy to self-renewal during homeostasis and repair. Cell. 2008;135:1118-29. https://doi.org/10.1016/j. cell.2008.10.048.

18. Cheng $\mathrm{T}$, et al. Hematopoietic stem cell quiescence maintained by p21cip1/waf1. Science. 2000;287:1804-8.

19. Pietras EM, Warr MR, Passegue E. Cell cycle regulation in hematopoietic stem cells. J. Cell Biol. 2011;195:709-20. https ://doi.org/10.1083/jcb.201102131.

20. Trumpp A, Essers M, Wilson A. Awakening dormant haematopoietic stem cells. Nat. Rev. Immunol. 2010;10:201-9. https:// doi.org/10.1038/nri2726.

21. Nakamura-Ishizu A, Takizawa H, Suda T. The analysis, roles and regulation of quiescence in hematopoietic stem cells. Development. 2014;141:4656-66. https://doi.org/10.1242/dev.106575.

22. Morrison SJ, Scadden DT. The bone marrow niche for haematopoietic stem cells. Nature. 2014;505:327-34. https://doi. org/10.1038/nature12984.

23. Zon LI. Intrinsic and extrinsic control of haematopoietic stem-cell self-renewal. Nature. 2008;453:306-13. https://doi. org/10.1038/nature07038.

24. Frenette PS, Pinho S, Lucas D, Scheiermann C. Mesenchymal stem cell: keystone of the hematopoietic stem cell niche and a stepping-stone for regenerative medicine. Annu Rev Immunol. 2013;31:285-316. https://doi.org/10.1146/annurev-immun ol-032712-095919.

25. Scadden DT. The stem-cell niche as an entity of action. Nature. 2006;441:1075-9. https://doi.org/10.1038/nature04957.
26. Watt FM, Hogan BL. Out of Eden: stem cells and their niches. Science. 2000;287:1427-30.

27. Ito K, Suda T. Metabolic requirements for the maintenance of self-renewing stem cells. Nat Rev Mol Cell Biol. 2014;15:24356. https://doi.org/10.1038/nrm3772.

28. Shyh-Chang N, Daley GQ, Cantley LC. Stem cell metabolism in tissue development and aging. Development. 2013;140:2535-47. https://doi.org/10.1242/dev.091777.

29. Chandel NS, Jasper H, Ho TT, Passegue E. Metabolic regulation of stem cell function in tissue homeostasis and organismal ageing. Nat Cell Biol. 2016;18:823-32. https://doi.org/10.1038/ ncb3385.

30. Ito K, Ito K. Metabolism and the control of cell fate decisions and stem cell renewal. Annu Rev Cell Dev Biol. 2016;32:399-409. https://doi.org/10.1146/annurev-cellbio-111315-125134.

31. Wilkinson AC, Yamazaki S. The hematopoietic stem cell diet. Int J Hematol. 2018. https://doi.org/10.1007/s12185-018-2451-1.

32. Anso E, et al. The mitochondrial respiratory chain is essential for haematopoietic stem cell function. Nat Cell Biol. 2017;19:61425. https://doi.org/10.1038/ncb3529.

33. Cabezas-Wallscheid $\mathrm{N}$, et al. Vitamin A-retinoic acid signaling regulates hematopoietic stem cell dormancy. Cell. 2017;169(819):807-23. https://doi.org/10.1016/j. cell.2017.04.018. e.

34. Orkin SH, Zon LI. Hematopoiesis: an evolving paradigm for stem cell biology. Cell. 2008;132:631-44. https://doi.org/10.1016/j. cell.2008.01.025.

35. Woolthuis CM, Park CY. Hematopoietic stem/progenitor cell commitment to the megakaryocyte lineage. Blood. 2016;127:1242-8. https://doi.org/10.1182/blood-2015-07-60794 5.

36. Dykstra B, et al. Long-term propagation of distinct hematopoietic differentiation programs in vivo. Cell Stem Cell. 2007;1:218-29. https://doi.org/10.1016/j.stem.2007.05.015.

37. Knapp D, et al. Single-cell analysis identifies a CD33(+) subset of human cord blood cells with high regenerative potential. Nat Cell Biol. 2018;20:710-20. https://doi.org/10.1038/s4155 6-018-0104-5.

38. Carrelha J, et al. Hierarchically related lineage-restricted fates of multipotent haematopoietic stem cells. Nature. 2018;554:106-11. https://doi.org/10.1038/nature25455.

39. Sanjuan-Pla A, et al. Platelet-biased stem cells reside at the apex of the haematopoietic stem-cell hierarchy. Nature. 2013;502:2326. https://doi.org/10.1038/nature12495.

40. Yamamoto R, et al. Clonal analysis unveils self-renewing lineage-restricted progenitors generated directly from hematopoietic stem cells. Cell. 2013;154:1112-26. https://doi.org/10.1016/j. cell.2013.08.007.

41. Yamamoto R, et al. Large-scale clonal analysis resolves aging of the mouse hematopoietic stem cell compartment. Cell Stem Cell. 2018;22:600-7 e604. https://doi.org/10.1016/j.stem.2018.03.013.

42. Suda T, Suda J, Ogawa M. Disparate differentiation in mouse hemopoietic colonies derived from paired progenitors. Proc Natl Acad Sci USA. 1984;81:2520-4.

43. Ito K, Ito K. Hematopoietic stem cell fate through metabolic control. Exp Hematol. 2018;64:1-11. https://doi.org/10.1016/j. exphem.2018.05.005.

44. Osawa M, Hanada K, Hamada H, Nakauchi H. Long-term lymphohematopoietic reconstitution by a single CD34-low/negative hematopoietic stem cell. Science. 1996;273:242-5.

45. Benveniste P, Cantin C, Hyam D, Iscove NN. Hematopoietic stem cells engraft in mice with absolute efficiency. Nat Immunol. 2003;4:708-13. https://doi.org/10.1038/ni940.

46. Gazit R, et al. Fgd5 identifies hematopoietic stem cells in the murine bone marrow. J Exp Med. 2014;211:1315-31. https:// doi.org/10.1084/jem.20130428. 
47. Acar M, et al. Deep imaging of bone marrow shows nondividing stem cells are mainly perisinusoidal. Nature. 2015;526:126-30. https://doi.org/10.1038/nature15250.

48. Challen GA, Boles NC, Chambers SM, Goodell MA. Distinct hematopoietic stem cell subtypes are differentially regulated by TGF-beta1. Cell Stem Cell. 2010;6:265-78. https://doi. org/10.1016/j.stem.2010.02.002.

49. Chen JY, et al. Hoxb5 marks long-term haematopoietic stem cells and reveals a homogenous perivascular niche. Nature. 2016;530:223-7. https://doi.org/10.1038/nature16943.

50. Ito K, et al. Self-renewal of a purified Tie2+ hematopoietic stem cell population relies on mitochondrial clearance. Science. 2016;354:1156-60. https://doi.org/10.1126/science.aaf55 30 .

51. Turcotte R, et al. Image-guided transplantation of single cells in the bone marrow of live animals. Sci Rep. 2017;7:3875. https://doi.org/10.1038/s41598-017-02896-6.

52. Walter D, et al. Exit from dormancy provokes DNA-damage-induced attrition in haematopoietic stem cells. Nature. 2015;520:549-52. https://doi.org/10.1038/nature14131.

53. Katajisto P, et al. Stem cells. Asymmetric apportioning of aged mitochondria between daughter cells is required for stemness. Science. 2015;348:340-3. https://doi.org/10.1126/ science. 1260384.

54. Youle RJ, Narendra DP. Mechanisms of mitophagy. Nat Rev Mol Cell Biol. 2011;12:9-14. https://doi.org/10.1038/nrm30 28.

55. Simsek T, et al. The distinct metabolic profile of hematopoietic stem cells reflects their location in a hypoxic niche. Cell Stem Cell. 2010;7:380-90. https://doi.org/10.1016/j.stem.2010.07.011.

56. Takubo K, et al. Regulation of glycolysis by Pdk functions as a metabolic checkpoint for cell cycle quiescence in hematopoietic stem cells. Cell Stem Cell. 2013;12:49-61. https://doi. org/10.1016/j.stem.2012.10.011.

57. de Almeida MJ, Luchsinger LL, Corrigan DJ, Williams LJ, Snoeck HW. Dye-independent methods reveal elevated mitochondrial mass in hematopoietic stem cells. Cell Stem Cell. 2017;21(e724):725-9. https://doi.org/10.1016/j. stem.2017.11.002.

58. Vannini N, et al. Specification of haematopoietic stem cell fate via modulation of mitochondrial activity. Nat Commun. 2016;7:13125. https://doi.org/10.1038/ncomms 13125.

59. Romero-Moya D, et al. Cord blood-derived CD34+ hematopoietic cells with low mitochondrial mass are enriched in hematopoietic repopulating stem cell function. Haematologica. 2013;98:1022-9. https://doi.org/10.3324/haematol.2012.079244.

60. Gan B, et al. Lkb1 regulates quiescence and metabolic homeostasis of haematopoietic stem cells. Nature. 2010;468:701-4. https ://doi.org/10.1038/nature09595.

61. Gurumurthy S, et al. The Lkb1 metabolic sensor maintains haematopoietic stem cell survival. Nature. 2010;468:659-63. https ://doi.org/10.1038/nature09572.

62. Nakada D, Saunders TL, Morrison SJ. Lkb1 regulates cell cycle and energy metabolism in haematopoietic stem cells. Nature. 2010;468:653-8. https://doi.org/10.1038/nature09571.

63. Chen C, et al. TSC-mTOR maintains quiescence and function of hematopoietic stem cells by repressing mitochondrial biogenesis and reactive oxygen species. J Exp Med. 2008;205:2397-408. https://doi.org/10.1084/jem.20081297.

64. Xiao N, et al. Hematopoietic stem cells lacking Ott1 display aspects associated with aging and are unable to maintain quiescence during proliferative stress. Blood. 2012;119:4898-907. https://doi.org/10.1182/blood-2012-01-403089.

65. Suda T, Takubo K, Semenza GL. Metabolic regulation of hematopoietic stem cells in the hypoxic niche. Cell Stem Cell. 2011;9:298-310. https://doi.org/10.1016/j.stem.2011.09.010.
66. Rossi DJ, Jamieson CH, Weissman IL. Stems cells and the pathways to aging and cancer. Cell. 2008;132:681-96. https:// doi.org/10.1016/j.cell.2008.01.036.

67. Ito K, et al. Reactive oxygen species act through p38 MAPK to limit the lifespan of hematopoietic stem cells. Nat Med. 2006;12:446-51. https://doi.org/10.1038/nm1388.

68. Ito K, et al. Regulation of oxidative stress by ATM is required for self-renewal of haematopoietic stem cells. Nature. 2004;431:997-1002. https://doi.org/10.1038/nature02989.

69. Miyamoto K, et al. Foxo3a is essential for maintenance of the hematopoietic stem cell pool. Cell Stem Cell. 2007;1:101-12. https://doi.org/10.1016/j.stem.2007.02.001.

70. Liang R, Ghaffari S, Mitochondria. and FOXO3 in stem cell homeostasis, a window into hematopoietic stem cell fate determination. J Bioenerg Biomembr. 2017;49:343-6. https://doi. org/10.1007/s10863-017-9719-7.

71. Testa U, Labbaye C, Castelli G, Pelosi E. Oxidative stress and hypoxia in normal and leukemic stem cells. Exp Hematol. 2016;44:540-60. https://doi.org/10.1016/j.exphe m.2016.04.012.

72. Maryanovich M, et al. An MTCH2 pathway repressing mitochondria metabolism regulates haematopoietic stem cell fate. Nat Commun. 2015;6:7901. https://doi.org/10.1038/ncomms8901.

73. Mohrin M, et al. Stem cell aging. A mitochondrial UPR-mediated metabolic checkpoint regulates hematopoietic stem cell aging. Science. 2015;347:1374-7. https://doi.org/10.1126/scien ce.aaa2361

74. Mohrin M, Widjaja A, Liu Y, Luo H, Chen D. The mitochondrial unfolded protein response is activated upon hematopoietic stem cell exit from quiescence. Aging Cell. 2018. https://doi. org/10.1111/acel.12756.

75. Yu WM, et al. Metabolic regulation by the mitochondrial phosphatase PTPMT1 is required for hematopoietic stem cell differentiation. Cell Stem Cell. 2013;12:62-74. https://doi.org/10.1016/j. stem.2012.11.022.

76. Raffel S, et al. BCAT1 restricts alphaKG levels in AML stem cells leading to IDHmut-like DNA hypermethylation. Nature. 2017;551:384-8. https://doi.org/10.1038/nature24294.

77. Tefferi A, et al. IDH1 and IDH2 mutation studies in 1473 patients with chronic-, fibrotic- or blast-phase essential thrombocythemia, polycythemia vera or myelofibrosis. Leukemia. 2010;24:1302-9. https://doi.org/10.1038/leu.2010.113.

78. Figueroa ME, et al. Leukemic IDH1 and IDH2 mutations result in a hypermethylation phenotype, disrupt TET2 function, and impair hematopoietic differentiation. Cancer Cell. 2010;18:55367. https://doi.org/10.1016/j.ccr.2010.11.015.

79. Agathocleous M, et al. Ascorbate regulates haematopoietic stem cell function and leukaemogenesis. Nature. 2017;549:476-81. https://doi.org/10.1038/nature23876.

80. Cimmino L, et al. Restoration of TET2 function blocks aberrant self-renewal and leukemia progression. Cell. 2017;170:107995e1020. https://doi.org/10.1016/j.cell.2017.07.032.

81. Aguilo F, et al. Prdm16 is a physiologic regulator of hematopoietic stem cells. Blood. 2011;117:5057-66. https://doi. org/10.1182/blood-2010-08-300145.

82. Luchsinger LL, de Almeida MJ, Corrigan DJ, Mumau M, Snoeck HW. Mitofusin 2 maintains haematopoietic stem cells with extensive lymphoid potential. Nature. 2016;529:528-31. https://doi. org/10.1038/nature 16500 .

83. Umemoto T, Hashimoto M, Matsumura T, Nakamura-Ishizu A, Suda T. $\mathrm{Ca}(2+)$-mitochondria axis drives cell division in hematopoietic stem cells. J Exp Med. 2018;215:2097-113. https://doi. org/10.1084/jem.20180421.

84. He C, Klionsky DJ. Regulation mechanisms and signaling pathways of autophagy. Annu Rev Genet. 2009;43:67-93. https://doi. org/10.1146/annurev-genet-102808-114910. 
85. Galluzzi L, Pietrocola F, Levine B, Kroemer G. Metabolic control of autophagy. Cell. 2014;159:1263-76. https://doi.org/10.1016/j. cell.2014.11.006.

86. Ueno T, Komatsu M. Autophagy in the liver: functions in health and disease. Nat Rev Gastroenterol Hepatol. 2017;14:170-84. https://doi.org/10.1038/nrgastro.2016.185.

87. Warr MR, et al. FOXO3A directs a protective autophagy program in haematopoietic stem cells. Nature. 2013;494:323-7. https:// doi.org/10.1038/nature11895.

88. Doulatov S, et al. Drug discovery for Diamond-Blackfan anemia using reprogrammed hematopoietic progenitors. Sci Transl Med. 2017. https://doi.org/10.1126/scitranslmed.aah5645.

89. Liu F, et al. FIP200 is required for the cell-autonomous maintenance of fetal hematopoietic stem cells. Blood. 2010;116:480614. https://doi.org/10.1182/blood-2010-06-288589.

90. Mortensen M, et al. Loss of autophagy in erythroid cells leads to defective removal of mitochondria and severe anemia in vivo. Proc Natl Acad Sci USA. 2010;107:832-7. https://doi. org/10.1073/pnas.0913170107.

91. Riffelmacher T, Simon AK. Mechanistic roles of autophagy in hematopoietic differentiation. FEBS J. 2017;284:1008-20. https ://doi.org/10.1111/febs.13962.

92. Ho TT, et al. Autophagy maintains the metabolism and function of young and old stem cells. Nature. 2017;543:205-10. https:// doi.org/10.1038/nature21388.

93. Jin G, et al. Atad3a suppresses Pink1-dependent mitophagy to maintain homeostasis of hematopoietic progenitor cells. Nat Immunol. 2018;19:29-40. https://doi.org/10.1038/s4159 0-017-0002-1.

94. Zimdahl B, et al. Lis1 regulates asymmetric division in hematopoietic stem cells and in leukemia. Nat Genet. 2014;46:245-52. https://doi.org/10.1038/ng.2889.

95. Ito $\mathrm{T}$, et al. Regulation of myeloid leukaemia by the cell-fate determinant Musashi. Nature. 2010;466:765-8. https://doi. org/10.1038/nature09171.

96. Jaiswal S, et al. Age-related clonal hematopoiesis associated with adverse outcomes. N Engl J Med. 2014;371:2488-98. https://doi. org/10.1056/NEJMoa1408617.

97. Genovese G, et al. Clonal hematopoiesis and blood-cancer risk inferred from blood DNA sequence. N Engl J Med. 2014;371:2477-87. https://doi.org/10.1056/NEJMoa1409405.

98. Sperling AS, Gibson CJ, Ebert BL. The genetics of myelodysplastic syndrome: from clonal haematopoiesis to secondary leukaemia. Nat Rev Cancer. 2017;17:5-19. https://doi.org/10.1038/ nrc.2016.112.

99. Steensma DP, et al. Clonal hematopoiesis of indeterminate potential and its distinction from myelodysplastic syndromes. Blood. 2015;126:9-16. https://doi.org/10.1182/blood-2015-03-631747.

100. Papaemmanuil E, et al. Clinical and biological implications of driver mutations in myelodysplastic syndromes. Blood. 2013;122:3616-27. https://doi.org/10.1182/blood-2013-08518886 (quiz 3699).

101. Corces MR, Chang HY, Majeti R. Preleukemic hematopoietic stem cells in human acute myeloid leukemia. Front Oncol. 2017;7:263. https://doi.org/10.3389/fonc.2017.00263.
102. Sarkozy C, et al. Outcome of older patients with acute myeloid leukemia in first relapse. Am J Hematol. 2013;88:758-64. https ://doi.org/10.1002/ajh.23498.

103. Bonnet D, Dick JE. Human acute myeloid leukemia is organized as a hierarchy that originates from a primitive hematopoietic cell. Nat Med. 1997;3:730-7.

104. Huntly BJ, Gilliland DG. Cancer biology: summing up cancer stem cells. Nature. 2005;435:1169-70. https://doi. org/10.1038/4351169a.

105. Shlush LI, et al. Tracing the origins of relapse in acute myeloid leukaemia to stem cells. Nature. 2017;547:104-8. https://doi. org/10.1038/nature22993.

106. Morrison SJ, Kimble J. Asymmetric and symmetric stem-cell divisions in development and cancer. Nature. 2006;441:1068-74. https://doi.org/10.1038/nature04956.

107. Kharas MG, et al. Musashi-2 regulates normal hematopoiesis and promotes aggressive myeloid leukemia. Nat Med. 2010;16:9038. https://doi.org/10.1038/nm.2187.

108. Wu M, et al. Imaging hematopoietic precursor division in real time. Cell Stem Cell. 2007;1:541-54. https://doi.org/10.1016/j. stem.2007.08.009.

109. Ward PS, et al. The common feature of leukemia-associated IDH1 and IDH2 mutations is a neomorphic enzyme activity converting alpha-ketoglutarate to 2-hydroxyglutarate. Cancer Cell. 2010;17:225-34. https://doi.org/10.1016/j.ccr.2010.01.020.

110. Garcia-Prat L, Sousa-Victor P, Munoz-Canoves P. Proteostatic and metabolic control of stemness. Cell Stem Cell. 2017;20:593608. https://doi.org/10.1016/j.stem.2017.04.011.

111. Jiang Y, Nakada D. Cell intrinsic and extrinsic regulation of leukemia cell metabolism. Int J Hematol. 2016;103:607-16. https:// doi.org/10.1007/s12185-016-1958-6.

112. Agathocleous M, et al. Ascorbate regulates haematopoietic stem cell function and leukaemogenesis. Nature. 2017. https://doi. org/10.1038/nature23876.

113. Wilkinson AC, Morita M, Nakauchia H, Yamazaki S. Branchedchain amino acid depletion conditions bone marrow for hematopoietic stem cell transplantation avoiding amino acid imbalance-associated toxicity. Exp Hematol. 2018;63(11):12-6. https ://doi.org/10.1016/j.exphem.2018.04.004. e.

114. Hattori A, et al. Cancer progression by reprogrammed BCAA metabolism in myeloid leukaemia. Nature. 2017;545:500-4. https://doi.org/10.1038/nature22314.

115. Sumitomo Y, et al. Cytoprotective autophagy maintains leukemia-initiating cells in murine myeloid leukemia. Blood. 2016;128:1614-24. https://doi.org/10.1182/blood-2015-12684696.

116. Duarte D, et al. Inhibition of endosteal vascular niche remodeling rescues hematopoietic stem cell loss in AML. Cell Stem Cell. 2018;22:64-77.e66. https://doi.org/10.1016/j.stem.2017.11.006.

117. Hawkins ED, et al. T-cell acute leukaemia exhibits dynamic interactions with bone marrow microenvironments. Nature. 2016;538:518-22. https://doi.org/10.1038/nature19801. 